\title{
LA FORMACIÓN UNIVERSITARIA EN IBEROAMÉRICA DESDE LO VIRTUAL, COMO ENTORNO PARA LA CALIDAD PEDAGÓGICA EN LA EDUCACIÓN SUPERIOR
}

\author{
Por: Bernardita Elisa Pupiales Ruano ${ }^{1}$ - Luis Alexander de Fonseca Tinoca ${ }^{2}$
}

\begin{abstract}
RESUMEN
El presente artículo muestra los resultados de la reflexión sobre el tema $L a$ Formación Universitaria en Iberoamérica desde lo virtual, como entorno para la calidad pedagógica en la Educación Superior, que gira en torno a la formación virtual como un espacio posible para la calidad de la formación superior. En él se concluye que para lograr la calidad a partir de la formación virtual es necesario posicionarnos como investigadores permanentes, aprendices de nuevas formas y espacios de comunicación e investigación, y abrir el debate sobre el tema de cómo relacionar la teoría y la práctica en aras de un saber menos complejo, compartimentado y teórico. La idea es que a partir de la reflexión, construyamos y propiciemos espacios y estrategias para interrelacionar estos saberes que constituyen la piedra angular de la formación de los profesionales en los diversos ámbitos del saber.

Para llevar a cabo la investigación se escogieron 120 artículos sobre el tema, publicados en revistas nacionales e internacionales. Para elegirlos se hizo una primera selección, a partir del criterio; debían ser artículos resultados de investigaciones sobre el tema o propuestas de innovación a través de las TIC, igualmente debían ser experiencias validadas en la práctica. El artículo se desarrolla bajo tres perspectivas: Hacia un entorno de calidad en la formación virtual, las Estrategias que determinan la calidad en la formación universitaria, Elearning y las herramientas digitales como medios para la calidad, conclusiones, debate y bibliografía.
\end{abstract}

1 Doctora en Formación Inicial y Permanente e Innovación Educativa. Doctorada por la Universidad Complutense de Madrid, España. Post doctorada. Universidad de Lisboa, Portugal. Docente Universidad del Tolima, Ibagué, Colombia.

2 Doctor en Ciencias de la Educación. Universidad de Austin, Texas. Docente Asistente Universidad de Lisboa, Portugal.

Fecha de recepción: 1 de marzo de 2014 - Fecha de aprobación definitiva: 18 de abril de 2014 
Los testimonios que acompañan el texto, fueron extraídos de entrevistas aplicadas a docentes que utilizan las Tics, para el desarrollo de las asignaturas en aulas de instituciones universitarias presenciales de Portugal.

Palabras clave: Formación, entorno virtual, calidad, mediación, proceso.

Clasificación JEL: 121

\title{
UNIVERSITY EDUCATION IN LATIN AMERICA FROM THE VIRTUAL ENVIRONMENT FOR QUALITY TEACHING IN HIGHER EDUCATION
}

\author{
By: Bernardita Elisa Pupiales Ruano - Luis Alexander de Fonseca Tinoca
}

\begin{abstract}
This paper presents the results of the research on the topic The University Education in Latin America from the virtual environment for innovation in teaching. The reflection revolves around virtual training as a process for quality in higher education. It is concluded that to achieve quality in virtual formation is necessary to relate the theory and practice for the sake of a less complex knowledge, subdivided and theoretical, providing new opportunities and strategies for both knowledge to interrelate.

To carry out the research 120 articles were selected on the subject, published in national and international journals. To choose them, a first selection was made from the criterion: the articles should be results of research on the subject or proposed innovation through ICT. Also they should be validated in practice experiences. The paper develops three perspectives: Towards a quality environment in virtual training, the strategies that determine the quality of university education, E-learning and digital tools as a means of quality, conclusions, discussion and bibliography.
\end{abstract}

Key words: Training, virtual environment, quality mediation process.

JEL Classification: 121. 
Bernardita Elisa Pupiales Ruano - Luis Alexander de Fonseca Tinoca

La formación universitaria en iberoamérica desde lo virtual, como entorno para la calidad pedagógica en la educación superior

Las nuevas tecnologías y los medios digitales plantean nuevas posibilidades educativas, dando paso a lo que se conoce como elearning. Sin embargo, contar con estos medios no garantiza sin más el conseguir los mejores resultados de aprendizaje, pues también se requiere la implantación paralela de nuevos modelos pedagógicos. Estos modelos afectarán tanto al diseño de los materiales educativos en formato digital, como al modo de utilización de los mismos en el aula.

Fermoso, G. y Pedrero, E. (2009: 1)

\section{INTRODUCCIÓN}

La universidad, creada en el Medioevo como respuesta a la necesidad social de establecer un espacio para el debate y el pensamiento, desde sus inicios no fue ajena a los cuestionamientos de los pensadores contemporáneos de su momento. Entonces los filósofos y los humanistas propuestos en el objetivo de desentrañar el pensamiento de la Escolástica centraron su interés en el estudio de los Universales, lo cual se convirtió en la entrada por la puerta grande hacia el conocimiento (siglo XI) y en la mejor posibilidad para ampliar la perspectiva del saber. Conocer el pensamiento de aquéllos que habían profundizado en la reflexión respecto a los temas trascendentales de la época posibilitó ampliar la visión respecto a la fe y la razón, la teología, la iglesia, el poder civil, el hombre y la ciencia.

Este fue un espacio que se estableció desde entonces con matices globales, el cual abrió paso a los grandes debates filosóficos que marcan un antes y un después de la educación. Desde entonces la educación logró uno de sus objetivos que hoy, ya en el siglo XXI, se establece como uno de los principios que rigen la ciencia: la universalidad del conocimiento. Según, Moreno A. J. y Albaizar F. A., La universidad moderna, la universidad de la sociedad del conocimiento, la universidad motor del desarrollo económico y social, debe ser globalmente competitiva y localmente comprometida (2009: 83).

En la llamada sociedad de la información y del conocimiento la tendencia es a acceder cada vez a un mayor y diverso conocimiento. Internet ofrece un sinnúmero de posibilidades e involucra cada vez a un mayor número de personas, sin embargo aún no es posible para un buen porcentaje de la población. Se puede afirmar que casi el 100\% de los estudiantes de educación superior de Iberoamérica conviven con las tecnologías, el acceso a ellas es un sendero paralelo con la formación profesional, este hecho es inherente a los estudiantes cuyo proceso de formación universitaria inició después de los 90s. Para quienes la biblioteca estuvo contenida en la Red, ellos son nativos digitales, por lo tanto el colectivo asume las mudanzas de internet con la familiaridad que tiene el abrir o cerrar un libro, por lo tanto es un deber del docente iniciar la mudanza de una pedagogía basada en el uso de material físico o en papel para asumir la complementación del proceso a través de la utilización de las TICs. Para Domingo, C. (2010); Como profesores del siglo XXI tenemos alumnos que son nativos digitales, aunque una gran mayoría del profesorado seamos inmigrantes digitales. A la vez, como profesores profesionales (nativos o inmigrantes digitales) debemos responder a las necesidades de una sociedad tecnológica, de una sociedad en red, y de una escuela que está inmersa en la 
sociedad de la información y la comunicación (2010: 171). El uso de internet surge en los años 90 y da paso a la web 1, cuya limitación fue la falta de interactividad, luego la web 2.0 y posibilita la interacción "in situ", y da paso a una comunicación y/o relación directa con el otro, es una especie de magia, que posibilita contacto; voz e imagen, en esta medida la atracción es excepcional. El carácter flexible de la formación virtual, la convierte en una posibilidad para acceder al conocimiento sin limitaciones de tiempo, ni de espacio. Varias son las propuestas de formación, tanto mixta, como completamente virtual que se ciernen a lo largo y ancho de la formación tanto en Europa, como en América, esta es quizá una forma de democratizar el conocimiento a la cual tienen acceso los sectores menos favorecidos de la población, que por diversos motivos no han logrado acceder al aula universitaria para complementar dicha formación.

Una de las características principales de la formación virtual es la accesibilidad con un importante valor añadido al evento formativo. Varias de las transformaciones más importantes en este ámbito se llevan a cabo desde las universidades europeas y norteamericanas, como también neozelandesas. Entre las experiencia interesantes, la universidad americana logra a través de la red acercar el conocimiento a un buen porcentaje de la población; en el 2008, cerca del 25\% del total de los alumnos universitarios estaban inscritos en cursos totalmente online (Allen \& Seaman, 2010), dicho ámbito registró una tasa de crecimiento del $21 \%$ en matrículas, el aumento en general fue de aproximadamente un $2 \%$ (Allen \& Seaman, 2010).

Según el Ministerio de Educación Nacional de Colombia, la educación virtual, también llamada "educación en línea", hace referencia al desarrollo de programas de formación que tienen como escenario de enseñanza y aprendizaje el ciberespacio, en el cual no es necesario que se dé un encuentro cara a cara entre el profesor y estudiante, sin embargo es posible establecer una relación interpersonal de carácter educativo.

Sin embargo, dicha formación no se desarrolla a partir de únicamente llevar la información a la plataforma, la formación debe suscitar una serie de interrogantes en cuanto a la forma, y al modelo pedagógico con el cual se lleva a cabo. Una formación de calidad depende en gran medida de las estrategias pedagógicas aplicadas en el proceso y la formación profesional tiene la responsabilidad, entre otros aspectos, de posibilitar a través del currículo el desarrollo de competencias y habilidades inherentes a cada profesión. En esta medida una de las necesidades prioritarias en el ámbito de la formación virtual es disponer de estrategias que logren la aplicación del conocimiento teórico en la práctica. Este es quizá el interrogante central y el motivo de reflexión para lograr la calidad en la formación virtual.

La práctica del conocimiento teórico estimula la reflexión y la producción del mismo, ya que en el aula universitaria el conocimiento es el resultado de la interacción desde la perspectiva del docente y de la del estudiante y la práctica, "in situ", hace posible que ese tipo de saber se inserte en la realidad del quehacer profesional. Calidad, no es cantidad, el proceso formativo en la educación superior implica la formación del espíritu científico, la preservación de la cultura y el fortalecimiento del pensamiento crítico y creativo, entre otros. 
Bernardita Elisa Pupiales Ruano - Luis Alexander de Fonseca Tinoca

La formación universitaria en iberoamérica desde lo virtual, como entorno para la calidad pedagógica en la educación superior

Según el CNA a la Educación Superior le compete formar intelectuales capaces de responder a las necesidades sociales con la herramienta de los conocimientos actuales, y de pensar y proponer alternativas viables de desarrollo que sean coherentes con los ideales establecidos a lo largo de la historia y expresados en las normas legales. La educación superior es responsable de la formación de profesionales capaces de crear conocimiento y de proponer nuevas formas de análisis y nuevas herramientas y relaciones de trabajo en todas las áreas. La educación superior es, en síntesis y como dice la Constitución, un servicio público que tiene una función social estratégica y que por tanto debe ser prestado con la mayor calidad posible.

\section{(Constitución Política de Colombia).}

El interrogante gira en torno a ¿cómo se construye la relación teoría-práctica en la formación virtual?, ¿existen otras posibilidades pedagógicas para dar respuesta a este tipo de interrogante?, ¿en qué medida la práctica o el conocimiento práctico perdió vigencia en la formación de los profesionales en los diversos ámbitos del saber? Cabe anotar que en los programas de formación de licenciados, la intervención en el aula, es una posibilidad para desarrollar competencias generales y específicas, saberes válidos, desde distintos espacios para todos los ámbitos de la formación.

Generalmente se concibe que la práctica posibilita el conocimiento práctico, para Domingo, C. M. y Fuentes, A. M. el proporcionar un entorno educativo en el que puedan desarrollarse, de manera efectiva nuestros alumnos está en manos de los educadores (2010: 171). En esta medida, toda acción formativa, deberá incluir estrategias para que no se quede en un saber sobre el papel, o teórico es necesario proporcionar espacios para desarrollar las habilidades requeridas.

Según el Ministerio de Educación de Colombia, los parámetros para medir calidad en programas de formación universitaria giran en torno a:

- La extensión social, la generación de nuevo conocimiento, la investigación, la construcción de la pertinencia social a través del currículo.

- La generación de proyectos en el ámbito académico y tecnológico con resultados de acción continúa.

- La pertinencia del currículo para lograr la relación entre las competencias básicas, genéricas, específicas y transversales.

- La interdisciplinariedad.

- La vinculación de estudiantes en proyectos de estimulación, creación y desarrollo intelectual y cultural.

- El fortalecimiento de Competencias comunicativas generales.

- La formación en cuanto a habilidades para buscar, clasificar, seleccionar y contrastar información, para realizar trabajo colaborativo.

- La adopción de las equivalencias de trabajo docente y estudiantil a créditos.

- Dedicación del personal académico a la proyección social.

En cuanto a los programas ofrecidos en la metodología a distancia, o programas a través de tecnologías de la información y la comunicación, además de los parámetros anteriores el MEN los insta a la búsqueda de la calidad a través de llevar a 
cabo la selección de los docentes, aplicando instrumentos y mecanismos precisos (encuestas, entrevistas, demostraciones virtuales, seminarios de inducción etc.) para establecer la idoneidad del candidato en cuanto a aptitudes y condiciones técnicas mínimas requeridas para trabajar con estrategias e instrumentos propios de estas metodologías educativas, además para la evaluación de los estudiantes.

... tengo que conocer y utilizar las nuevas TIC a través de otros contextos, especialmente en relación con la investigación y ejemplos de trabajos prácticos en equipos (como ocurrió, por ejemplo, en residencias de artistas en la que participé con los miembros de los laboratorios $A Z$ - hackerspaces LCD y altLab XDA). Docente Facultad Bellas Artes U. Lisboa (2013).

En el proceso formativo, no sólo en la universidad, igualmente en otras etapas de la formación, es el docente quien interviene directamente en el diseño y planificación de las actividades que afectan la transformación del estudiante, sin embargo que el docente proponga y el estudiante cumpla no es la panacea de la formación; para Michavila, F. y Parejo, J.: ...se perfilan puntos de mejora en relación con el cambio cultural deseable para mejorar la participación estudiantil en todos los niveles y ámbitos del sistema universitario (...) La participación entendida como competencia cívica, debe ser un contenido formativo aprendido por los estudiantes en su paso por la universidad con la finalidad de convertirse en ciudadanos activos, comprometidos y responsables con la sociedad $(2008,85)$.

En el 2011 se llevó a cabo el Informe de Cisco, sobre tecnología en el mundo, para determinar cómo y en qué medida la próxima generación de trabajadores se verá influenciada por este medio. El informe concluye, entre otros aspectos que: "Uno de cada tres estudiantes universitarios y jóvenes profesionales considera Internet tan importante como las necesidades humanas básicas, tales como agua, aire, alimento y refugio, además, que internet ha dejado en segundo plano los medios de comunicación tradicional, como la TV”. Fuente, Informe CISCO (2011). Dos tercios de los estudiantes (66\%) y más de la mitad de los empleados (58\%) citan a un dispositivo móvil (equipo portátil, smartphone, tablet) como la tecnología más importante en sus vidas. Informe Cisco (2011).

... las estrategias más aceptadas por los estudiantes fueron la publicación del proceso y los resultados de los ejercicios en línea, explorando nuevas áreas y enfoques, por ejemplo, la computación física y programación creativa. Docente Facultad Bellas Artes U. Lisboa (2013).

Menos de 1 de cada 10 estudiantes universitarios (6\%) y de los empleados (8\%) respondió que la televisión constituye la tecnología más importante en sus vidas, y se espera que esta tendencia continúe. En general, más de uno de cada cuatro estudiantes universitarios (27\%) asumió que mantenerse actualizado en Facebook era más importante que salir a fiestas, tener citas, escuchar música o estar con los amigos (Informe Cisco, 2011).

En esta medida hacer uso del entorno virtual presupone una opción para disminuir las desigualdades de partida en cuanto al acceso a la educación de un buen porcentaje de sujetos; sin embargo el evento requiere una reflexión crítica sobre 
Bernardita Elisa Pupiales Ruano - Luis Alexander de Fonseca Tinoca

La formación universitaria en iberoamérica desde lo virtual, como entorno para la calidad pedagógica en la educación superior

la responsabilidad social que acontece a la acción formativa; al respecto, García, L. (2008) comenta: En los países emergentes económicamente, los beneficios de las tecnologías de la información TIC, sólo han sido aprovechados por las organizaciones para su inserción en el mercado global. En contraste, en las comunidades de estos países donde se desarrollan procesos de localización arraigada, las TIC no han sido un factor de crecimiento. (...) La globalización económica y tecnológica sólo ha beneficiado a corporaciones haciendo más extensa la brecha económica y digital con las comunidades (2008, 64).

\section{ESTRATEGIAS QUE DETERMINAN LA CALIDAD EN LA FORMACIÓN PROFESIONAL UNIVERSITARIA}

Los objetivos deben redactarse de forma que todos los estudiantes tengan claro qué se espera de ellos al finalizar el proceso de enseñanza/aprendizaje; recalcar lo fundamental, lo que se evaluará.

García Cabrera, L. y Otros (2010: 83)

Una formación virtual pertinente, relaciona los objetivos de la formación superior con las necesidades del medio y logra la comprensión de los problemas cotidianos del entorno para buscar soluciones a los mismos a través de la investigación. En esta medida la formación universitaria se percibe como un bien de carácter sociocultural. En el caso de la educación colombiana se hace imperativo formar teniendo en cuenta las demandas actuales de la sociedad; la diversidad cultural, la construcción de una sociedad justa y equitativa a través de posibilidades laborales. Las instituciones educativas deben propender por abrir nuevos espacios de intervención en cada ámbito, es necesario que se cree conciencia sobre la necesidad de formar para la investigación permanente y la proposición de nuevas miradas a los ámbitos de desempeño. Es preciso orientar al estudiante a buscar caminos posibles para dar respuesta a los interrogantes de cada ámbito de del saber a través de la investigación

Para Berry (1991) uno de los pilares para el desarrollo de estrategias de respuestas a los nuevos desafíos en educación superior es precisamente el cuerpo docente y una de las formas de dar respuesta a estos desafíos reside en su formación pedagógica profesional. En el sistema de educación superior colombiano existen tres aspectos que determinan la calidad de la formación universitaria, entre ellos se encuentran los resultados de las pruebas ECAES, los resultados de investigación y la productividad intelectual de los docentes; la coherencia entre los programas que ofrecen las instituciones educativas y las necesidades del medio, además de la articulación de los proyectos de extensión social universitaria. Dicha calidad está dada por la relación entre currículos pertinentes, la consolidación de escenarios propicios para el aprendizaje y las prácticas profesionales acordes a los ámbitos específicos del saber, entre otros; a efectos de redefinir el concepto de educación universitaria, De la Orden propone, tener en cuenta los siguientes factores: Las expectativas y necesidades sociales, como funcionalidad, las metas y objetivos de 
educación universitaria, definidas como eficacia o efectividad y los productos de la Universidad, como eficiencia (1997: 4).

La formación requiere tener en cuenta una serie de elementos sin los cuales no es posible el acceso y la motivación por el conocimiento, y es precisamente el docente/investigador quien debe asumir una relación total, para hacer posible este tipo de interacción. Para Tello L. y otros: Podemos afirmar que la calidad de la educación determinará si en el futuro los jóvenes tendrán las habilidades necesarias para incrementar significativamente su competitividad en una economía global cada vez más integrada. En este sentido, se requerirán no sólo más y mejores habilidades específicas, sino también la capacidad de participar activamente en la adquisición de nuevos conocimientos para enfrentar nuevas problemáticas (Tello, L. y otros, 2010).

Brew e Bound (1996), enfatizan algunas de las posibilidades de formación de docentes de educación superior destacando:

- Programas de formación íntimamente ligados a las prioridades de la institución;

- Programas específicos para el desempeño de papeles específicos;

- Introducción, en el diseño de los programas, de conocimiento sobre el aprendizaje de adultos y sobre estrategias de cambio;

- Programación de los momentos de formación, en alternancia con la actividad docente del día-a-día;

- Programas de respuesta más general, más flexibles, sobre todo abarcando temas concretos, aplicables a las actividades de los docentes;

- Programas que puedan implicar diferentes parceros - o formando, o formador y un supervisor en "terreno";

- Formas alternativas de evaluación, con la utilización de un conjunto variado de instrumentos;

- Atribución de créditos de formación y asunción de estos créditos en progresión de la carrera.

La universidad debe formar para pensar, para interactuar, para crear ciencia y tecnología y para proyectar soluciones desde la teoría y la práctica; y en la misma medida para el desarrollo humano. Actualmente, se espera de las Instituciones de Enseñanza Superior integren las necesidades de un mercado de trabajo cada vez más complejo y diversificado en su oferta pedagógica, de manera que formen ciudadanos autónomos, y profesionales críticos (Tinoca, Pereira e Oliveira, 2014). Por su parte, la Ley 30 de 1992, exhorta al reconocimiento de procesos de calidad en las instituciones de educación superior, lo cual incide en un mayor esfuerzo por mejorar los resultados educativos. Y describe a la educación superior como un proceso permanente que posibilita el desarrollo de las potencialidades del ser humano de manera integral, se realiza con posterioridad a la educación media o secundaria y tiene por objeto el pleno desarrollo de los alumnos y su formación académica o profesional (MEN, 1992: 99). Una de las recomendaciones en el proceso 
Bernardita Elisa Pupiales Ruano - Luis Alexander de Fonseca Tinoca

La formación universitaria en iberoamérica desde lo virtual, como entorno para la calidad pedagógica en la educación superior

del mejoramiento y Aseguramiento de la Calidad es la articulación con el proceso de evaluación permanente de la institución, lo cual posibilita conocer los aciertos y las desviaciones del proyecto académico, como valor agregado al proceso. Collis, B y Moonen T, afirman: para que la flexibilidad pase de estar anclada en las opciones de uso logístico y personal a formar parte de aspectos más fundamentales de la educación superior, seguirá siendo necesario aplicar incentivos estratégicos y dar apoyo adecuado al cambio participativo y pedagógico (2011:18).

Finalmente es necesario un proceso de articulación desde los contenidos, ya que deben ser trabajados de manera que les posibiliten formar desde la reflexiónacción y a partir del estímulo de la creatividad, avanzando hacia la formación de un profesional capaz de buscar soluciones a los problemas que rodean el ámbito de actuación. En este sentido, para Gimeno S.: el aprendizaje como indagación y la creatividad acompañada de la crítica se erigen como las competencias claves del ciudadano para poder afrontar la incertidumbre y la super-complejidad de su contexto (2008: 76). Uno de los problemas y fenómenos que debería afrontar y resolver con ahínco la institución universitaria es la formación pedagógica de los docentes, y más aún si estos no han iniciado a trasegar por el ámbito de la investigación y/o la formación en el ambiente virtual. En este sentido en las universidades se encuentra que un $95 \%$ de docentes han egresado de programas distintos a los de licenciatura, poseen conocimientos específicos, pero carecen de conocimientos pedagógicos, la intervención se caracteriza por una ínfima participación del estudiante, el cual repite y transcribe las intervenciones en el aula, se vuelve incapaz de explorar otras formas para acceder y producir conocimiento.

En cuanto a este referente encontramos a Bausela, H.: entre las habilidades de un profesor motivador están estimular las iniciativas y creatividad de su alumnado, mantener la expectación sobre lo que sucederá en la clase, intentar conectar con el centro de interés del alumnado (2005: 2). La actuación del docente universitario del siglo XXI deberá tener en cuenta las características de una generación, que si bien no ha nacido en la era digital, la explora y permanece en actuación a través de ella, con lo cual la utilización de las TIC's se convierte en una estrategia ineludible a la cual no es posible renunciar, ya sea por desconocer el potencial que suscita o por la falta de formación de los docentes para adoptarla en su quehacer pedagógico; para Domingo, C. M y Fuertes A. M, en un estudio realizado sobre la incorporación de las Tecnologías de la Información y la Comunicación en el aula, concluyen en cuanto a los docentes: mayoritariamente, un 90\% destaca que las TIC suponen una renovación metodológica innovadora que propicia un aumento de la motivación y participación de los estudiantes, que facilita su comprensión y el aprendizaje en general, que proporciona nuevos recursos educativos, y que aumenta la satisfacción, motivación y autoestima del docente. Y que en menor medida, un $60 \%$ valora que facilita el trabajo colaborativo, que puede aumentar la autonomía de los estudiantes, y que facilita su evaluación y autoevaluación (2010: 174).

Estudios realizados sobre la forma cómo han logrado los docentes realizar intervenciones de calidad, dan cuenta de lograr el puente entre el estudiante y el conocimiento a través de todo aquello planeado estratégicamente para lograr no sólo los objetivos de la asignatura, sino la motivación permanente por el conocimiento. Para Bain, K. (2007), un docente excelente es aquel que primordialmente ayuda a 
sus estudiantes a aprender, y además consigue influir positivamente en su forma de pensar, actuar y sentir. En esta medida mejorar la calidad de la intervención pedagógica implica un cambio de postura frente a aquello que se enseña, y lo que realmente el estudiante necesita interiorizar; frente a esto afirman De Vicente R. y otros (2010): toda práctica educativa obedece a una concepción de la materia que se enseña y del conocimiento que de ella se tenga.

La excelencia docente implica la preparación de clase con un alto nivel de profundización y con óptimo diseño de estrategias que medien entre el estudiante y el conocimiento. Bain, K. dice sobre un docente excelente conocen bien sus disciplinas, y son académicos, artistas o científicos en activo y expertos, (...) tienen un sentido inusualmente agudo de la historia de sus disciplinas, incluyendo las controversias que se han agitado en ellas, y esa comprensión parece que les ayuda a reflexionar de manera especialmente profunda sobre la naturaleza del pensamiento en sus campos; de igual manera y utilizan "esa capacidad para pensar sobre su propio razonamiento -lo que llamamos meta cognición”, se dan cuenta en dónde es fácil que las personas encuentren dificultades a la hora de avanzar en su propia comprensión. (2007: 36).

Se destaca que en la formación a través del entorno virtual la investigación es aún incipiente no sólo en Colombia, sino en Suramérica, con lo cual, aún no emergen los inconvenientes del proceso; sin embargo, precisamente por ser un ámbito en construcción, es necesario lograr la complementariedad del evento educativo para fortalecer y profundizar el acceso al conocimiento en las diversas disciplinas, iniciando un proceso de investigación a través de la plataforma virtual. El conocimiento específico constituye una parte considerable de la formación en cada saber, la formación interdisciplinaria deberá integrar los saberes proporcionando esa visión general/particular proporcionando un saber menos compartimentado y real del profesional.

Cuando enseño cursos anuales, lanzado casi dos prácticos y uno teórico. Después de Bolonia, no hay tiempo suficiente para el seguimiento y debate adecuado, por lo que propongo a los ejercicios prácticos que están implicados referentes teóricos presentados en clase".

Docente Facultad Bellas Artes U. Lisboa (2013).

Para terminar, asumir la docencia en la educación superior presupone llevarla a cabo a partir de una perspectiva científica, para lo cual el docente deberá implicarse en un proceso de investigación permanente y de desarrollo profesional que le permita conocer y profundizar el conocimiento hacia:

- El descubrimiento del potencial del estudiante mediante la exploración del mismo a través de diversas actividades pedagógicas.

- La apertura de nuevos espacios para ofrecer -otros- ambientes pedagógicos.

- Motivar para y hacia la producción de bienes para la sociedad.

- Posibilitar el conocimiento de estrategias para el análisis interdisciplinario del saber. 
- Estimular la creatividad, a partir de la búsqueda de soluciones a los problemas científicos desde una perspectiva divergente.

- Ofrecer espacios para llevar a cabo y estimular el trabajo cooperativo y en equipo.

- Propiciar espacios para fortalecer la libre expresión, el análisis crítico, y la reflexión en la acción, en aras de formar profesionales capaces de afrontar una sociedad en permanente cambio.

- Incentivar la lectoescritura investigativa como posibilidad para acceder a nuevo conocimiento.

- Posibilitar la accesibilidad al conocimiento de los estudiantes con discapacidad

- Estimular la participación de los estudiantes en grupos de investigación consolidados.

- Motivar la capacidad de asombro y la capacidad creativa.

- Incentivar la articulación del proyecto educativo con el trabajo práctico aplicando dicho conocimiento en el entorno más cercano.

Las experiencias de innovación a través de las TIC han acompañado la evolución social y tecnológica, y se reconoce que el paso por la universidad es tratar de explorar demasiado, por lo que los alumnos son muy receptivos al uso de las TIC en general - ya que no forma parte sus expectativas de aprendizaje. Docente Facultad Bellas Artes U. Lisboa (2013).

\section{ELEARNING Y LAS HERRAMIENTAS DIGITALES COMO MEDIOS PARA LA CALIDAD}

Los objetivos deben redactarse de forma que todos los estudiantes tengan claro qué se espera de ellos al finalizar el proceso de enseñanza/aprendizaje; recalcar lo fundamental, lo que se evaluará.

García Cabrera, L. y Otros (2010: 83).

La formación profesional requiere el desarrollo y fortalecimiento de una serie de habilidades intrínsecas que determinan la calidad, para la puesta en escena de dichos profesionales. En esta medida es necesario construir una perspectiva de la evaluación para el desarrollo profesional, la cual deberá integrar los saberes desde diversas ópticas; Pupiales-Rueda (2011) expresa: un docente no es únicamente quien planifica e imparte la asignatura, es en suma un pensador de la misma, de los procesos que conllevan a la motivación de los estudiantes por aprender y lograr un conocimiento profundo y de indagación permanente.

La evaluación, por su parte, como eje complementario del proceso, es quizá uno de los eventos educativos, que antes que proporcionar información sobre cuánto aprendió el estudiante, da cuenta sobre cómo y en qué medida el docente es coherente tanto en la teoría desde la cual desarrolla la intervención docente, como en la práctica de la misma. No sólo es necesario presentar y diseñar el componente de evaluación, sino también asumirlo pedagógicamente. 
... de alguna manera, las referencias que se dan en la clase están disponibles en línea para que los estudiantes exploren de forma individual, todo lo que pueden y les interesa, por otro lado, también puede comentar en línea, hablar y hacer preguntas. Excepcionalmente, hacer preguntas a través de correo electrónico.

Docente Facultad Bellas Artes U. Lisboa (2013).

La evaluación forma y transforma al estudiante, ella marca las pautas que luego el estudiante aplicará en el entorno laboral en el cual desarrolla la intervención profesional. Al respecto afirman Paoloni V, y Rinaldo C.: La visión de la tarea académica como contexto de aprendizaje se ha complejizado, sus características se interpretan como dinámicas y no estáticas, en permanente interacción con factores personales y contextuales, y no deterministas en sus efectos potenciales, lo que significa que los rasgos de una tarea no necesariamente redundarán en beneficios motivacionales en los estudiantes por el sólo hecho de cumplir con lo teóricamente estipulado (2009: 4). No es fácil asumir una evaluación desde la perspectiva del compromiso por el saber, desde la reflexión en la acción, y no únicamente para identificar si se ha retenido la teoría, es necesario lograr una evaluación desde la puesta en marcha del conocimiento en el espacio de la práctica. García Cabrera, L y Otros: En un entorno virtual la evaluación debe estar basada más en la realización de trabajos, actividades y proyectos que en pruebas teóricas. Debe quedar claro lo que se va a evaluar, tipos de evaluaciones y con qué peso (2010: 83).

Tinoca, Pereira y Oliveira (2014) refuerzan igualmente la necesidad de alinear las estrategias de evaluación implementadas en estos nuevos contextos virtuales/ digitales con esta nueva cultura de aprendizaje centrada en el estudiante, donde la evaluación se pretende sobre todo formativa y a partir de una perspectiva edumétrica (Dierick y Dochy, 2001). La evaluación por su parte implica la relación con el proceso, ya que a partir de ella el estudiante se hace una idea de aquello trascendental para fortalecer, y que es indispensable conocer en cada ámbito del saber. De ella se aprende tanto en lo didáctico como en lo humano. Para Fernández Pérez, M. (1998) solo se evaluará lo que estuvo en el proceso, no lo que no estuvo, si no fue posible percibirlo, menos aún evaluarlo. La evaluación no sólo construye, también proyecta. Para Rendón U., M.: No se trata de evaluar por evaluar, sino más bien de evaluar en coherencia con un planteamiento curricular, es decir, de orientación formativa. Se requiere hacer posible que lo evaluativo se integre realmente a la práctica pedagógica y deje de ser una especie de "rueda suelta". Es más: las prácticas de la evaluación del aprendizaje deben responder a las características de los saberes disciplinares para contribuir a conformar una sólida estructura de los mismos, lo cual implica, como ya se mencionó, tener la posibilidad de un manejo flexible y abierto de la evaluación (2009).

... Los estudiantes deben usar las computadoras para realizar los ejercicios / proyectos. Acompañar a las obras que publican en el blog y galería de trabajos, respondo a sus comentarios, publicar directrices línea.

Docente Facultad Bellas Artes U. Lisboa (2013). 
De esta manera es necesario crear las condiciones y ofrecer un ambiente para el debate, y para la reinterpretación de los valores/cuestiones vitales de la vida académica, al igual que para la subsistencia del planeta, para lo cual se debe aportar conocimiento, a través de la reflexión sobre la acción. Para García Cabrera, L. y Otros: La evaluación en entornos virtuales estará basada más en la aplicación de lo aprendido, en el desarrollo de destrezas y habilidades sociales, que en la memorización. Además, habrá que facilitarle la información de contacto de todas las personas involucradas en la acción formativa, tanto docentes como técnicas, que podrán ayudarle a resolver cualquier problema relacionado con el uso de la plataforma de Docencia Virtual (2010).

... excepcionalmente, algunos estudiantes tienen cierta inhibición / compleja en cuanto a su uso en un principio, pero luego comenzaron a hacer el trabajo son entusiastas y pueden tener más confianza en sus habilidades como usos menos convencionales de las TIC a las nuevas situaciones. Docente Facultad Bellas Artes U. Lisboa (2013).

Las instituciones que se acogen a los sistemas internos de evaluación de la calidad crecen, se fortalecen y generan un mayor compromiso de todos los involucrados en el proceso. El Foro Nacional sobre Evaluación en la Educación Superior-Colombia 2008, identifica diversas perspectivas que inducen a repensar el evento, más como espacio formativo, que utilitario para medir conocimientos; por su parte, Rendón Uribe, M. y Otros (2009), identifican que existe la necesidad de practicar una evaluación mucho más participativa, más "actuante", puesto que se desarrolla para un público homogéneo y en un buen porcentaje constituido por población de adultos.

... tomé una actitud exploratoria, las referencias para lanzar retos y enfoques no convencionales y ejercicios que promueven la investigación, el desarrollo de intercambio de ideas y alternativas.

Docente Facultad Bellas Artes U. Lisboa (2013).

Uno de los cuestionamientos de la universidad del Siglo XXI, es la deserción estudiantil, el tema que es quizá una de las tareas pendientes, tanto para los países europeos, como para sur América. Este hecho presupone una pérdida económica tanto para el estudiante, como para las familias y la sociedad. Al respecto, García Cabrera, L. y Otros están de acuerdo en, que es necesario un acercamiento entre el docente y el discente, evitar la sensación de soledad, de incertidumbre a la que se puede enfrentar el alumno (2010). La deserción de los estudiantes se genera por varios aspectos, uno de ellos es la falta de una orientación profesional adecuada en la educación secundaria, lo cual genera equivocación en cuanto a la escogencia de la carrera, muchos tienen la vocación, por la carrera pero carecen de las habilidades requeridas para el ámbito de formación escogido.

Por su parte, Miñano, P. y Castejón afirman que entre las variables cognitivomotivacionales sobre el rendimiento académico se encuentran La Inteligencia Gene- 
ral/aptitudes, Atribuciones causales, Auto concepto Académico, Metas y Estrategias de Aprendizaje (2008: 1). Otros estudiantes encuentran dificultades en el proceso derivado de las asignaturas troncales, como resultado del desconocimiento de un estilo de aprendizaje que responda a la necesidad personal o individual del o la estudiante, lo cual mejoraría si él o la docente profundiza en el conocimiento de los métodos didácticos de intervención/evaluación necesarios y adecuados para complementar un proceso educativo, con múltiples requerimientos ya sea desde lo científico, intelectual, y/o humano.

Cabanach, G. y Otros, por su parte asumen que un bajo sentido de eficacia personal aparece frecuentemente vinculado con elevados niveles de ansiedad y sintomatología propia del estrés y que aquellos individuos que tienen elevados niveles de autoeficacia mantienen niveles de malestar significativamente inferiores (2010: 75).

En este sentido es necesario dar respuestas a los interrogantes de la nueva etapa, identificando núcleos problémicos y posibilitando un acompañamiento adecuado de los estudiantes con el objetivo de que el espacio universitario favorezca ambientes para motivarlos hacia el crecimiento personal y el desarrollo humano. Es necesario el compromiso de la comunidad educativa en general para acompañar al estudiante en el proceso de adaptación a la vida universitaria y así disminuir el índice de deserción. Es necesaria la reflexión sobre la responsabilidad del docente frente al fracaso del estudiante, dado que es su responsabilidad proporcionar el acceso al conocimiento. Este hecho implica el conocimiento de la didáctica, el fortalecimiento de la sensibilidad pedagógico/creativa, entre otros. Para Rosario, P. y otros los alumnos con más fracaso escolar se sienten menos eficaces para autoregular su aprendizaje (2012:37).

Porque yo uso regularmente las TICs en la actualidad, pero he tenido talleres de introducción a la programación y el uso creativo de la plataforma Moodle, esto a través de UL eLearning.

Docente Facultad Bellas Artes U. Lisboa (2013).

Es imperativo un trabajo de acompañamiento que posibilite la cercanía con el estudiante, tanto desde el aula como a través de los estamentos encargados de este tipo de actividades dentro del campus universitario. Al respecto afirman Rosario, P. y otros: La magnitud del fenómeno y las obvias implicaciones sociales sugieren que este no es solo un tema prioritario de los investigadores del área educativa, sino también de toda la sociedad (2012, Ibíd.). Está visto que en un 80\% la actividad formativa depende de la actividad de docencia, por lo tanto, es prioritario crear incentivos orientados a fortalecer a los docentes con resultados educativos muy buenos, encaminando la intervención hacia la excelencia académica, como el valor agregado en la cual está en juego no solo la formación de profesionales, sino el futuro de la nación y el aporte a la construcción del saber desde una perspectiva pedagógica, científica y humana.

Por su parte, Collins B. y Monen Jef, afirman: entendemos por flexibilidad la opción de ofrecer a los estudiantes la posibilidad de elegir cómo, qué, dónde, cuándo y con quién participan en las actividades de aprendizaje (2010:1). Esto corrobora que es el docente quien tiene relación directa con el estudiante, relación que se 
Bernardita Elisa Pupiales Ruano - Luis Alexander de Fonseca Tinoca

La formación universitaria en iberoamérica desde lo virtual, como entorno para la calidad pedagógica en la educación superior

convierte en la razón de ser y el centro del evento educativo y de ella dependen en gran medida los resultados del aprendizaje. Así lo confirman Marín, D. y Maldonado, B.: la combinación del binomio TIC-Educación Superior nos lleva a pensar en tres beneficios que tal matrimonio puede aportar a la práctica universitaria, por un lado la innovación, por otro la apertura del sistema al exterior y en último lugar la posibilidad de desarrollar comunidades virtuales de aprendizaje entre docentes universitario (2010:121). El referente de calidad, como eje transversal del proceso de formación profesional deberá considerar prioritaria la investigación en torno a aspectos que tienen que ver con la intervención pedagógica, el acompañamiento, la guía y la orientación del estudiante teniendo en cuenta que para muchos estudiantes la formación profesional es quizá el único espacio de cercanía con el ámbito educativo formal, con lo cual es imperativo formar en un ambiente de calidad, sea para la adquisición de competencias, conocimiento, habilidades tanto hacia la ciencia, como también para la formación permanente.

Conviene no olvidar que de la calidad de la Enseñanza universitaria dependen muchas cosas y todas ellas importantes.

La propia excelencia humana está puesta en juego en ella y, por consiguiente, el futuro -también el próximo- de las personas y de la entera sociedad.

Polaino Lorente, A. (2006)

\section{CONCLUSIONES}

La formación universitaria, presencial o virtual, deberá asumir la calidad como eje transversal del proceso para lograr un alto grado de motivación, no sólo para superar la calificación mínima de la asignatura, sino como la antesala hacia la formación permanente.

En el contexto actual promovido por la nueva cultura de aprendizaje, sustentado por la expansión del uso de las nuevas tecnologías, es imperativa la reconceptualización de la evaluación y sus funciones, de los modelos e instrumentos de evaluación, con el propósito de asegurar su calidad.

La formación a partir de la plataforma virtual, deberá ofrecer formación a través de procesos de investigación sobre el quehacer pedagógico, ya que es necesario conocer: ¿cómo aprenden los estudiantes?, ¿cómo logran retener ese conocimiento a lo largo de la etapa de formación?, y ¿cómo se convierte en conocimiento real? El saber pedagógico, como el específico requieren abordarse a partir de un proceso dinámico, pertinente, innovador, creativo y flexible.

Es necesario que el proceso se oriente a fortalecer y desarrollar la capacidad crítica, creativa, pedagógica y de interpretación del entorno del estudiante, en aras de formar la conciencia social.

En la fusión pedagogía y virtualidad, aparte de la formación para el conocimiento, lo que realmente importa, es aquello que se logra transformar dentro del estudiante, no solo en el ámbito de la cultura profesional, también de la percepción de desarrollo humano. 
La evaluación debe ser validada y contrastada desde diversas ópticas, es necesario que el conocimiento se lleve a la práctica y en un entorno real y que el proceso formativo tenga en cuenta que existen múltiples inteligencias, como diversas formas de aprender dependiendo de las primeras.

Toda acción formativa lleva implícitos aspectos de desarrollo cognitivo y ético, en el caso de la Formación Virtual, la tecnología ofrece posibilidades para el fortalecimiento de las dimensiones del ser, acción que lleva implícita una perspectiva de desarrollo psicosocial.

\section{DEBATE}

Todo proceso de formación debe generar una relación con el medio, de esta manera es posible hablar de una educación eficaz, en la medida en que ésta es capaz de generar la práctica del conocimiento teórico en un espacio real.

La docencia universitaria implica la coherencia entre el discurso del docente y la intervención pedagógica en el aula. Tanto el proceso formativo como evaluativo deberán constituir espacios para la formación.

La educación superior debe prever una formación que además motive al estudiante por aplicar dicho conocimiento con un alto nivel de calidad, generando una cultura profesional para y a partir de la calidad.

Tanto el proceso de formación, como de evaluación deben ir en concordancia con las habilidades y competencias que se desea desarrollar y fortalecer.

Para formar en armonía con las necesidades del medio, es necesario reinterpretar la acción pedagógica, formar implica conocer el camino, y a la vez producir cambios necesarios en una generación nacida en la era digital.

Los cambios o las mudanzas del trabajo docente deberán posibilitarle nuevas perspectivas hacia la acción de enseñar a aprender.

La acción de formar, es también una posibilidad de interrelación entre el docente, el estudiante y el conocimiento, lo cual implica la interacción desde la teoría y hacia una práctica real del conocimiento.

La formación virtual debe tener en cuenta la cultura del entorno en el cual se encuentra imbricada, además de diseñar el contenido según el público al cual va dirigida para adoptar un proceso pertinente tanto en lo social como en lo académico.

La formación superior debe generar espacios para el desarrollo profesional docente, para que a partir de la construcción de equipos de trabajo colaborativo, los docentes realicen observación, reflexión y análisis sobre la intervención en el aula, lo cual conlleva a desarrollar y fortalecer competencias pedagógicas, tendientes a hacer posible una formación superior, que adopta la calidad como eje transversal del proceso. 


\section{REFERENCIAS}

ANDION, Mauricio (2007). Sobre la calidad de la educación superior: una visión cualitativa. En Revista Reencuentro. Universidad Autónoma Metropolitana, Xochimilco. 83/92. Recuperado en: http://redalyc.uaemex. $\mathrm{mx} / \mathrm{src} /$ inicio/ArtPdfRed.jsp?iCve=34005011. 7 marzo de 2008.

BAUSELA, H. y Otros (2005). Ansiedad ante los exámenes: evaluación e Intervención psicopedagógica. En EDUCERE. Universidad de los Andes. Venezuela. No 031. Vol. 9. 553/558. Recuperado en: http://redalyc.uaemex.mx/pdf/356/35603117.pdf. 2 de agosto de 2008.

BELLIDO, J. (2005). Motivar en el aula: El Arte de hacer que hagan. Aljibe: España.

BENEDITO, I. y ANTOLI, V. (1993). Formación permanente del profesorado universitario: reflexiones y perspectivas. III Jornadas Nacionales de Didáctica Universitaria "Evaluación y Desarrollo Profesional", pp. 229-259. Ed. Servicio de Publicaciones de la Universidad de Las Palmas de Gran Canaria.

BERRY, E.; FILBECK, M.; ROTHSTEIN-FISH, C. \& SALTMAN, H. (1991). Implementing classroom research in a state university: a developmental process. In T. Angelo (Ed.). Classroom research: Early lessons from success. New directions for teaching and learning, Vol. 46, pp. 91-104. San Francisco: Jossey-Bass.

BREW, A., \& BOUND (1996). Preparing for new academic roles: a holistic approach to development. The International Journal for Academic Development. 1 (2), 17-25.

CABANACH y Otros (2010). Las creencias motivacionales como factor protector del estrés en estudiantes universitarios. European Journal of Education and Psychology. Vol. 3, No 1.75/87. Recuperado en: http://redalyc. uaemex.mx/redalyc/pdf/1293/129313736006.pdf. 1 de marzo de 2012.

CAMARGO-ESCOBAR M. y PARDO-ADAMES, R. (2008). Competencias docentes de Profesores de pregrado: diseño y validación de un instrumento de evaluación. Vol. 7. No 2. Universidad Católica de Colombia. Recuperado en: clombia.vhttp://revistas.javeriana.edu.co/index.php/revPsycho/article/view/441.

CARRILLO, G. y Otros (2011). Impacto de las mediaciones didácticas interactivas en el aprendizaje de los estudiantes del modelo educativo transformemos educando en el departamento de Córdoba. En: Revista Investigación y Desarrollo. Bogotá, Colombia. Vol. 19, No. 2.

CAMPO CABAL, A. y Otros (2006). Entornos Virtuales en la Educación Superior. Consejo Nacional de Acreditación. Bogotá.

CONSEJO NACIONAL DE ACREDITACIÓN -CNA-. Acreditación de programas de pregrado. Ministerio de Educación Nacional de Colombia. Recuperado en: http://www.cna.gov.co/1741/article-186377.html. 12 de enero de 2008.

CARBONERO, M. y Otros (2011). El Profesor estratégico como favorecedor del clima de aula. En: European Journal of Education and Psychology. Vol. 4, No. 2.133/142. Recuperado en: http://www.ejep.es/index.php/ journal/article/view/133-142. 21 de marzo de 2012.

COLLINS, B. y Moonen J. (2011). Flexibilidad en la educación superior: revisión de expectativas. En: Comunicar No. 37, Vol. XIX, 2011, Revista Científica de Edu-comunicación, pp. 15-25. XXI Conferencia Iberoamericana de Educación. Asunción, Paraguay, 26 de septiembre de 2011

Declaración de la XXI Conferencia Iberoamericana de Educación. Asunción, Paraguay, 26 de septiembre de 2011. Recuperado en: http://www2.minedu.gob.pe/digesutp/formacioninicial/wp-descargas/2011/XXI_Conferencia_iberamericana_de_educacion.pdf. 5 de diciembre 2011.

DIERICK, S., \& DOCHY, F. J. R. C. (2001). New lines in edumetrics: new forms of assessment lead to new assessment criteria. Studies in Educational Evaluation, 27, 307-329.

DOMINGO, C. María y FUENTES, Martha (2010). Innovación Educativa: experimentar con las Tic y reflexionar sobre su uso. En: Pixel-Bit. Revista de Medios y Educación. No. 36. 171/180. Universidad Autónoma de Barcelona. Recuperado en: http://www.sav.us.es/pixelbit/pixelbit/articulos/n36/13.pdf. 10 de abril de 2010. 
ESCOBAR, Arteaga, N. (2011). La Educación mediada por la Tecnología. Fundación Universitaria Tecnológico Comfenalco, Cartagena de Indias, Colombia. (№118 Noviembre).

ESTEVEC, Frances. (2009). La cuestión universitaria. Boletín electrónico de la Cátedra UNESCO de Gestión Política Universitaria. Universidad Politécnica de Madrid. Recuperado en: http://www.lacuestionuniversitaria. upm.es/web/articulo.php?id_articulo=42. 15 de marzo de 2012.

FERNÁNDEZ PÉREZ, Miguel (1988). Evaluación y Cambio Educativo. Madrid: Morata.

FERMOSO, Ana; PEDRERO, Alberto (2009). Educar haciendo uso de las nuevas tecnologías y medios digitales. Algunas pautas básicas. Facultad de Informática. Universidad Pontificia de Salamanca. http://revistas.um.es/ red/article/view/87811/84541. Recuperado el 10 de diciembre de 2011.

GARCÍA CABRERA, Lina (2010). La calidad en la docencia virtual: la importancia de la guía de estudio. En: Revista de Medios y Educación, Universidad de Jaén. Dpto. de Informática, Dpto. de Pedagogía, Facultad de Humanidades y Ciencias de la Educación, Escuela Politécnica Superior de Jaén. (No. 37. pp. 77/92 Pixel-Bit). Recuperado en http://www.sav.us.es/pixelbit/pixelbit/articulos/n37/7.pdf. 5 de marzo de 2012.

GARCÍA, Cruz (2008). La brecha digital de las generaciones futuras. En: Revista Entelequia. Revista interdisciplinar (No. 8. 61/72). Recuperado en: http://www.eumed.net/entelequia/pdf/2008/e08a07.pdf. 10 de febrero de 2012.

GONZALES C., Olga y FLORES F., Manuel (1998). El trabajo docente. Enfoques innovadores para el diseño de un curso. México: Trillas.

GIL SERRA, Ana; ROCA, Pierre. Movilidad virtual, reto del aprendizaje de la educación superior en la Europa 2020. En: Revista de Educación a Distancia. Universidad de Almería (Número 26). Recuperado en: http://www. redalyc.org/src/inicio/ArtPdfRed.jsp?iCve=54718738001. El 23/12/2011.

MIÑANO, P. y CASTEJON, C. (2008). Capacidad predictiva de las variables cognitivo-motivacionales sobre rendimiento académico. En: Revista Electrónica de Motivación y Emoción (Reme Vol. XI. No 28).Recuperado el 8 de marzo de 2012, en http://reme.uji.es

Informe Cisco (2011). Sobre tecnología virtual. Recuperado en: http://www.cisco.com/en/US/netsol/ns1120/ index.html. 12 de diciembre de 2011.

MICHAVILA, F. y PAREJO, J. (2008). Políticas de participación estudiantil en el proceso de Bolonia. En: Revista de Educación. Universidad Politécnica de Madrid (Número Extraordinario, 2008) p. 85/118. Recuperado en: http://www.revistaeducacion.mec.es/re2008/re2008_05.pdf. 13 de marzo de 2012.

MINISTERIO DE EDUCACIÓN NACIONAL. El sistema de Educación Superior de Colombia. Ministerio de Educación Nacional. Recuperado en: http://www.cna.gov.co/1741/article-187279.html.

MARÍN, D. y MALDONADO, B. (2010). El alumnado universitario Cordobés y la plataforma virtual Moodle. En: Revista Pixel-Bit. Revista de Medios y Educación (No 38, julio-diciembre), pp. 121-128.

MORENO A. J. y ALBAIZAR F. A. (2009). La tercera misión de la universidad. En el Libro Blanco de la Universidad. Recuperado en: http://www.universidaddigital2010.es/portal/page/udf/inicio/publico. 23 marzo el 2012.

Revista de Educación a Distancia. Sección de Docencia Universitaria en la Sociedad del Conocimiento (Número 26, 2011). Consultado el [16/12/2011] en http://www.um.es/ead/reddusc/3/

ROYERO, Jaim. Contexto sociopolítico de la evaluación de la calidad. En: OEI-Revista Iberoamericana de Educación. http://www.unmsm.edu.pe/occaa/articulos/contexto.pdf. Recuperado el 6 de marzo de 2012.

ROSARIO, P. y otros. (2012). Autoeficacia y utilidad percibida como condiciones necesarias para un aprendizaje académico autorregulado. En: Revista Anales de Psicología. Universidad de Murcia (Vol. 28. No 1). Recuperado en: http://revistas.um.es/analesps/article/view/140502, 9 de marzo de 2012.

PADILLA BELTRÁN, J. y otros (2008). Creencias de los docentes acerca del uso de las tecnologías de información y comunicación. En: Revista Educación y Desarrollo Social. Bogotá, D.C. vol. 2, pp. 45-57.

PEÑALOSA, C. y Otros (2010). Modelo estratégico de comunicación educativa para entornos mixtos de aprendizaje: estudio piloto. En: Revista Pixel-Bit. Revista de Medios y Educación. Universidad Autónoma Metropolitana - Cuajimalca (No 37. 43/55). Recuperado el 3 de febrero de 2012. En: http://www.redalyc.org/src/inicio/ ArtPdfRed.jsp?iCve=16713079005. 
PAOLONI V. y RINALDO C. (2009). Motivación, tareas académicas y procesos de feedback. Un estudio comparativo entre alumnos universitarios. En: Revista Electrónica de Motivación y Emoción. Vol. XI. No 31. Recuperado en http://reme.uji.es/articulos/numero31/article9/texto.html. 20 de marzo de 2012.

PAOLONI, P. y otros. Procesos de retroalimentación en la autorregulación de recursos de aprendizaje. Explorando su potencial en el contexto de la universidad. En: Revista Docencia Universitaria en la Sociedad del Conocimiento.

PUPIALES Rueda, B. (2011). El Desarrollo del Pensamiento Pedagógico de los egresados de las Facultades de Educación en la ciudad de San Juan de Pasto, Colombia. En: Revista TENDENCIAS, Facultad de Ciencias Económicas y Administrativas. Universidad de Nariño. Vol. XII No 2, pp. 82-106.

QUINCHE, J. y GONZÁLEZ, Franci L. (2011). Entornos Virtuales 3D, Alternativa Pedagógica para el Fomento del Aprendizaje Colaborativo y Gestión del Conocimiento. En: RED - Revista de Educación a Distancia. Sección de docencia universitaria en la Sociedad del Conocimiento. Universidad Minuto de Dios (Número 3). Recuperado el 5 de abril de 2012 en http://www.um.es/ead/reddusc/3/

RAMA, Claudio y PARDO, José (2011). La Educación superior a distancia: miradas diversas desde Iberoamérica. Institución tecnológica virtual de Educación. Printed.

ROBALINO, C. y KORNER, A. (2006). Modelos Innovadores en la formación docente. Estudio de casos de modelos innovadores de la formación docente en América Latina. Recuperado. 1/486. en: http://www.oei.es/docentes/publicaciones/docentes/modelos_innovadores_formacion_inicial_docente.pdf. 13 de febrero de 2010.

RIVERA y Otros. (2010). La transmisión visual de la información como estímulo cognitivo de los procesos creativos. En: Revista Anales de Psicología. Universidad de Murcia. Vol. 26. No 2, pp. 227-237. Recuperado en: http://www.redalyc.org/src/inicio/ArtPdfRed.jsp?iCve=16713079005. 1 de marzo de 2012.

RED dedicada a enseñanza universitaria: la docencia en la sociedad del conocimiento, política y gestión. Número 3, 15 de mayo de 2011. Recuperado en: http://www.um.es/ead/reddusc/3/index.html.

RENDÓN U, M. (2011). Los estilos de enseñanza en la Universidad de Antioquia. En: Memorias del Primer Congreso Internacional en Procesos Pedagógicos. Un enfoque interdisciplinario. Universidad Sergio Arboleda. Colombia.

SYBILLE, R. y TOUCH, C. (2005). Tendencias IV: Universidades europeas puesta en práctica de Bolonia. Estudio de European University Association. Recuperado el 10 de agosto de 2011 en: http://eees.universia.es/documentos/asociacion-universidad-europea/

TELLO, L. y Otros (2010). Análisis de los servicios de la tecnología Web 2.0 aplicados a la educación. En: Revista No solo usabilidad. Revista multidisciplinar sobre diseño, personas y tecnologías.oleda (Vol. 4, 2009).

TORREZ, Z. (2009). La evaluación del aprendizaje en la Educación Superior: lecciones aprendidas y perspectivas a partir del año de la Evaluación. 197/210. En: Memorias del Primer Congreso Internacional en Procesos Pedagógicos. Un enfoque interdisciplinario. Universidad Sergio Arboleda (Vol. 4), pp. 197-210.

TINOCA, L., PEREIRA, A. \& OLIVEIRA, I. (2014). A Theoretical Framework for E-Assessment in Higher Education: Authenticity, Consistency, Transparency and Practicability. In Siran Mukerji and Purnedu Tripathi (Eds.) Handbook of Research on Transnational Higher Education Management. IGI Global.

TINOCA, L. (2012). Avaliação 2.0: novas tecnologias ao serviço da aprendizagem. In Iracema Cusati, Adilson Xavier da Silva e Luís Tinoca (orgs.) Perspectivas em Avaliação Educacional: conceitos, processos e estratégias. Brasil, Curitiba, PR: Editora CRV.

\section{GLOSARIO}

ECAES: Exámenes para evaluar el nivel de conocimiento de los estudiantes universitarios colombianos.

CNA: Consejo Nacional para la Acreditación y evaluación de la calidad de los Programas académicos universitarios.

CISCO: Informe que devela el incremento de tráfico IP que previsiblemente viajará por redes públicas y privadas, incluyendo Internet, redes IP gestionadas y el tráfico móvil generado por los consumidores y usuarios empresariales.

MEN: Ministerio de Educación Nacional. 